\title{
BMJ Open Prevalence and factors associated with fatigue in the Lausanne middle-aged population: a population-based, cross- sectional survey
}

\author{
Coralie Galland-Decker, ${ }^{\circledR}$ Pedro Marques-Vidal, Peter Vollenweider
}

To cite: Galland-

Decker C, Marques-Vidal P, Vollenweider $P$. Prevalence and factors associated with fatigue in the Lausanne middle-aged population: a population-based, crosssectional survey. BMJ Open 2019;9:e027070. doi:10.1136/ bmjopen-2018-027070

- Prepublication history and additional material for this paper are available online. To view these files, please visit the journal online (http://dx.doi org/10.1136/bmjopen-2018027070).

Received 04 October 2018 Revised 23 July 2019 Accepted 26 July 2019

\section{Check for updates}

(c) Author(s) (or their employer(s)) 2019. Re-use permitted under CC BY-NC. No commercial re-use. See rights and permissions. Published by BMJ.

Department of Medicine, Internal Medicine, Lausanne University Hospital and University of Lausanne, Lausanne, Switzerland

\section{Correspondence to}

Professor Pedro Marques-Vidal; pedro-manuel.marques-vidal@ chuv.ch

\section{ABSTRACT}

Objective To assess the prevalence and factors associated with fatigue in the general population. Design Population-based, cross-sectional survey performed between May 2014 and April 2017.

Setting General population of the city of Lausanne, Switzerland

Participants 2848 participants $(53.2 \%$ women, age range 45-86 years).

Primary outcome measure Prevalence of fatigue the previous week, defined as a score of $\geq 4$ using the Fatigue Severity Scale.

Results The prevalence of fatigue was $21.9 \%(95 \% \mathrm{Cl}$ $20.4 \%$ to $23.4 \%$ ) in the total sample. On bivariate analysis, participants with fatigue were younger, had a higher body mass index, a lower handgrip strength and lower ferritin levels. Participants with fatigue were more frequently women, had a lower educational level, presented more frequently with clinical insomnia, diabetes, anaemia, depression and low thyroid stimulating hormone (TSH) values, had a higher consumption of antihistamines, antidepressants and hypnotics, and rated more frequently their health as bad or very bad. Multivariable analysis showed that obesity (OR $1.40(95 \% \mathrm{Cl} 1.03$ to 1.91$))$, insomnia categories ( $p$ value for trend $<0.001$ ), depression (OR 3.26 (95\% Cl 2.38 to 4.46)), anaemia (OR 1.70 (95\% $\mathrm{Cl} 1.00$ to 2.89)) and low self-rated health status (p value for trend $<0.001$ ) were positively associated with fatigue, while older age ( $p$ value for trend 0.002 ) was negatively associated with fatigue. Conversely, no association was found for diabetes, TSH levels, antihistamines or hypnotics. Conclusion In a population-based sample aged 45-86, fatigue was present in one out of five subjects. Regarding clinical factors, sleep disturbances such as insomnia and sleep apnoea should be assessed first, followed by depression. Regarding biological factors, anaemia should be ruled out, while screening for hypothyroidism is not recommended as a first step. Sleep complaints and fatigue in older subjects are not due to ageing and should prompt identification of the underlying cause.

\section{INTRODUCTION}

Fatigue is usually defined as 'an unpleasant physical, cognitive and emotional symptom described as a tiredness not relieved by common strategies that restore energy'.
Strengths and limitations of this study

This study assessed the prevalence and factors associated with fatigue in a general population setting.

- A large panel of possible factors associated with fatigue were evaluated.

- A list of the most frequent determinants was established, facilitating aetiological search in clinical practice.

- The study was limited to subjects aged 45-86, so results do not apply to younger or older groups.

Fatigue varies in duration and intensity and reduces the ability to perform usual daily activities. ${ }^{1}$ Indeed, fatigue is a common symptom with prevalence rates varying between $4 \%$ and $45 \% .^{2-4}$ This tenfold range in prevalence rates is likely due to the different settings (ie, general practice $^{5}$ or workers $^{6}$ ) or the different methods used to assess fatigue. ${ }^{7}$

In healthy subjects, tiredness or sleepiness is a natural occurrence after physical or mental efforts and is usually relieved by rest, ${ }^{89}$ while fatigue is defined as extreme and persistent tiredness, weakness or exhaustion of mental and/or physical origin ${ }^{7}$ that is not relieved by rest. Fatigue is defined in duration as recent ( $<1$ month), prolonged (1-6 months) and chronic ( $>6$ months).${ }^{10}$ When unexplained, chronic fatigue can be considered either as a syndrome (characterised by severe, disabling fatigue and other symptoms, including musculoskeletal pain, sleep disturbance, impaired concentration and headaches) ${ }^{11}$ or as idiopathic (absence of other symptoms).

Fatigue is one of the most common complaints reported in primary care ${ }^{12}$ and is associated with a decreased quality of life and increased morbidity and mortality in the general population. ${ }^{13}$ Fatigue is a multidimensional concept, and several determinants have been proposed. Although a 
cause (somatic or psychiatric) is identifiable in two thirds of fatigue cases, one third of fatigue cases still have no specific diagnosis. ${ }^{10}$ The most frequent diagnoses associated with fatigue are viral or upper respiratory tract infection, iron deficiency anaemia, adverse effects of medication, depression or other mental disorders. ${ }^{14}$ Fatigue has also been associated with female sex,${ }^{815}$ older age $^{1617}$ and lower socioeconomic status, ${ }^{16}{ }^{17}$ although the association with the last two determinants were not found in some studies. ${ }^{8}{ }^{18}$ Importantly, most studies on fatigue have been conducted in selected populations, such as workers ${ }^{6}$ or general practice attendees. ${ }^{2518}$ To our knowledge, only two studies have assessed the prevalence of fatigue in the general population, ${ }^{8} 19$ and only a few have explored the determinants of fatigue in the general population. ${ }^{1315-172021}$ Furthermore, most studies focused on socioeconomic and disease determinants of fatigue, while information regarding the biological determinants (ie, anaemia or thyroid pathology) ${ }^{13}$ or the medications associated with fatigue is scarce. Moreover, to date, little is known about the prevalence of fatigue and its determinants in Switzerland.

Hence, this study aimed to examine the prevalence and the factors associated with fatigue in a population-based sample from the city of Lausanne, Switzerland.

\section{POPULATION AND METHODS \\ Study population}

The Cohorte Lausannoise (CoLaus) study is a population-based cohort exploring biological, genetic and environmental determinants of cardiovascular diseases. Detailed descriptions of the study design have been reported elsewhere. ${ }^{22}$ Briefly, a non-stratified random representative sample of the population of Lausanne was recruited between 2003 and 2006 using the following inclusion criteria: (1) aged between 35 and 75 years and (2) willingness to participate. The first follow-up was performed between April 2009 and September 2012, and the second follow-up between May 2014 and April 2017, 10.9 years on average after the baseline study. At both baseline and subsequent follow-ups, participants were invited to attend a clinical examination at the Lausanne University Hospital. Participants received a paper questionnaire at home, which they filled in prior to the clinical examination. During the clinical examination, a second questionnaire regarding personal and family history of cardiovascular disease and cardiovascular risk factors was applied. For more details, please consult https://www. colaus-psycolaus.ch/.

As fatigue was only assessed in the second follow-up, data from the second follow-up, which included 4881 of the initial 6773 participants recruited at baseline, were used. At the second follow-up, participants were aged $45-86$ years.

\section{Fatigue scale}

Fatigue severity during the previous week was assessed by the nine-item Fatigue Severity Scale (FSS) ${ }^{9}$ The FSS is one of the most commonly used fatigue questionnaires. It has been validated in a healthy population setting in German-speaking Switzerland, ${ }^{23}$ Portugal $^{24}$ and Norway. ${ }^{19}$ It is a simple, time-saving, self-administrated questionnaire allowing its use in large epidemiological studies and has a high test-retest reliability. ${ }^{7}$ The questionnaire is composed of nine questions; responses are graded using a Likert scale from 1 to 7 , where 1 indicates strong disagreement and 7 strong agreement. The final score is the mean value of the nine responses, and a score of $\geq 4$ is considered as having severe fatigue. This cut-off was initially proposed because $<5 \%$ of healthy controls rate their fatigue at that level, whereas $60 \%-90 \%$ of patients with medical disorders experience fatigue at or above this level. ${ }^{9}$ An example of the questionnaire in French is provided in online supplementary annex 1 and in English in online supplementary annex 2. To our knowledge, the French version of the FSS has not yet been validated in Switzerland. Still, the Cronbach's alpha for the questionnaire was 0.918 , suggesting an excellent internal consistency.

\section{Covariates}

Socioeconomic and lifestyle variables were collected using a self-administered questionnaire. Smoking status was categorised into never, former and current smoker. Educational level was collected at baseline and categorised as obligatory school, apprenticeship, high school/ college or university.

Insomnia was assessed using the Insomnia Severity Index (ISI). ${ }^{25}$ The questionnaire has 16 items evaluating the nature, severity and impact of insomnia over the last month, namely difficulties falling asleep, sleep maintenance problems, and early morning awakening, sleep dissatisfaction, interference of sleep disturbances with daytime functioning, noticeability of sleep problems by others and distress caused by the sleep difficulties. Responses range from 0 'Not at all' to 4 'Extremely'. Items were scaled 0-4 and then summed to obtain the global ISI score (range: 0-28). The questionnaire is provided in online supplementary annex 3 in French and in online supplementary annex 4 in English. Clinically significant insomnia was defined as an ISI score $\geq 15$ (moderate to severe intensity). ${ }^{25}$

Depression was assessed with the Center for Epidemiologic Studies-Depression, ${ }^{26}$ a 20 -item self-report instrument developed for research in the general population and is used to assess the severity of depressive symptoms over the past week on a 4-point scale. It was translated into French by Fuhrer and Rouillon. ${ }^{27}$ It has been used in other recent epidemiological studies assessing the link between depression and cardiovascular risk factors. ${ }^{28}$ The questionnaire is composed of 20 questions; responses are graded from 0 to 3 , where 0 indicates rarely or never (less than 1 day), and 4 most or all of the time (5-7 days per week). The final score is the sum of the 20 responses (possible range is $0-60$ ), and a score of $\geq 16$ is considered a risk for depression. 
Self-rated health was assessed by a single question where participants had to rate their current health status from five categories ranging from 'very bad' to 'very good'. As the number of participants rating their health as 'very bad' was very small, they were grouped with the participants who rated their health as 'bad'.

Body weight and height were measured with participants standing without shoes in light indoor clothing. Weight was measured in kilograms to the nearest $0.1 \mathrm{~kg}$ using a seca scale (seca, Hamburg, Germany). Height was measured to the nearest $5 \mathrm{~mm}$ using a seca height gauge (seca). Body mass index (BMI) was defined as weight $/$ height ${ }^{2}$ and categorised as underweight (BMI $\left.<18.5 \mathrm{~kg} / \mathrm{m}^{2}\right)$, normal $\left(18.5 \leq \mathrm{BMI}<25 \mathrm{~kg} / \mathrm{m}^{2}\right)$, overweight $\left(25 \leq \mathrm{BMI}<30 \mathrm{~kg} / \mathrm{m}^{2}\right)$ and obese $\left(\mathrm{BMI} \geq 30 \mathrm{~kg} / \mathrm{m}^{2}\right)$.

Grip strength was assessed using the Baseline Hydraulic Hand Dynamometer (Fabrication Enterprises, Elmsford, New York, USA) with the subject seated, shoulders adducted and neutrally rotated, elbow flexed at $90^{\circ}$, forearm in neutral position, and wrist between $0^{\circ}$ and $30^{\circ}$ of dorsiflexion. Three measurements were performed consecutively with the right hand, and the highest value (expressed in kilograms) was included in the analyses.

Caffeinated drink consumption was assessed by the question 'How many cups or cans of drinks containing caffeine (coffee, tea, coke or similar) do you drink per day?' with possible answers 'None', ' $1-3$ ', ' $4-6$ ' and ' 7 or more'.

Participants were asked to report all medications (prescribed or bought over the counter) they took during the last 6 months. Medications were coded using the WHO's Anatomical Therapeutic Chemical (ATC) classification (www.whocc.no/atc_ddd_index/). Antihistamines were defined as any ATC code beginning with 'R06'; antidepressants were defined as an ATC code beginning with 'N05BD' or 'N06AA' or 'N06AB' or 'N06AF' or 'N06AG' or 'N06AX' or 'N06CA'; and hypnotics were defined as any ATC code beginning with 'N05C'. Antihypertensive drugs were defined by asking the participants if they were taking drugs for hypertension.

Diabetes was defined by a fasting plasma glucose $\geq 7 \mathrm{mmol} / \mathrm{L}$ and/or the presence of an antidiabetic drug treatment (oral or insulin). Personal history of cardiovascular disease was assessed by asking the participant if he/ she had sustained a coronary event (myocardial infarction or angina pectoris) or a stroke.

Biological assays were performed by the Centre Hospitalier Universitaire Vaudois (CHUV) Clinical Laboratory on fresh blood samples within 2 hours of blood collection, and additional aliquots were stored at $-80^{\circ} \mathrm{C}$. All measurements were conducted in a Modular $\mathrm{P}$ apparatus (Roche Diagnostics, Switzerland). The following analytical procedures (with maximum interbatch and intrabatch coefficients of variation (CVs)) were used: high sensitive C-reactive protein (hs-CRP) by immunoassay and latex high sensitive $(4.6 \%-1.3 \%)$; transferrin by immunoassay $(1.8 \%-1.0 \%)$; and glucose by glucose dehydrogenase $(2.1 \%-1.0 \%)$. Ferritin was assessed by immunoturbidimetric method (Tina-quant 4th Generation, Roche Diagnostics, Switzerland) with a maximum intra-assay CV of $7.2 \%$ and a maximum interassay CV of $9.9 \%$. Thyroid stimulating hormone (TSH) and free $\mathrm{T}_{4}$ were assessed by chemiluminescence on a Cobas e602 device (Roche Diagnostics, Mannheim, Germany) with intrabatch CVs ranging between $1.1 \%$ and $3.0 \%$ for TSH and between $2.7 \%$ and $5 \%$ for free $\mathrm{T}_{4}$.

\section{Exclusion criteria}

Participants were excluded if they lacked (1) any answer to the fatigue questionnaire; (2) clinical data such as age, BMI, smoking, depression, insomnia or medications; (3) biological measures such as haemoglobin or thyroid hormones; and (4) socioeconomic data such as educational level.

\section{Patient and public involvement}

No patients or public were involved in this study design, conduct or analysis.

\section{Statistical analysis}

Statistical analysis was performed using Stata V.15.1 for Windows. Prevalence rates for fatigue were expressed as percentage and 95\% CI. Descriptive results were expressed as number of participants (percentage) for categorical variables or as average $\pm \mathrm{SD}$ for continuous variables. Bivariate analyses were performed using $\chi^{2}$ or Fisher's exact test for categorical variables and Student's t-test or Kruskal-Wallis test for continuous variables. All categorical variables significantly $(\mathrm{p}<0.05)$ associated with fatigue in the bivariate analysis were included in the multivariable analysis. Multivariable analysis was performed using analysis of variance or logistic regression with fatigue (dichotomised into yes/no) as dependent variable; results were expressed as multivariable-adjusted mean $\pm \mathrm{SE}$ for continuous variables or as OR and $95 \% \mathrm{CI}$ for categorical variables.

Sensitivity analyses were conducted using an FSS threshold of 5. Further, as the number of excluded participants was high, other sensitivity analyses were conducted by creating a propensity score for being excluded. ${ }^{29}$ The propensity score was computed using logistic regression, with exclusion (yes/no) as dependent variable and all variables significantly associated with exclusion as independent variables. A probability of exclusion was computed for each participant, and the inverse of the probability was used for weighting.

Statistical significance was assessed for a two-sided test with $\mathrm{p}<0.05$.

\section{RESULTS}

\section{Study population}

Of the 4881 participants in the second follow-up, 2848 (58.4\%) were retained for analysis. The reasons for exclusion are summarised in online supplementary figure 1 ; the most frequent reason was lack of data regarding 
Table 1 Bivariate and multivariable analyses of the continuous factors associated with fatigue as defined by a Fatigue Severity Scale score $\geq 4$ in the Cohorte Lausannoise (CoLaus) study, Lausanne, Switzerland, 2014-2017

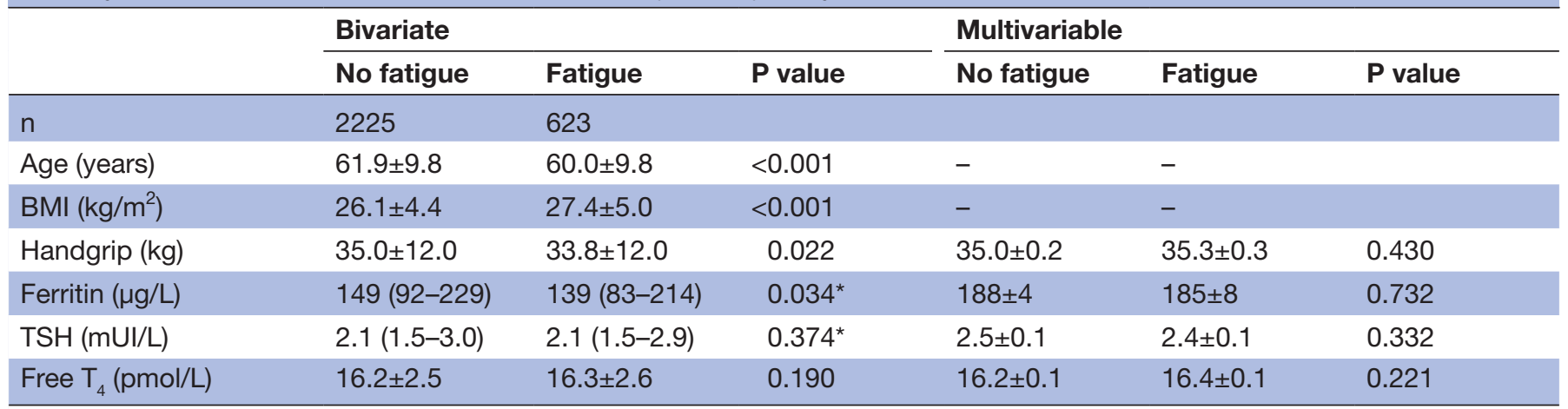

Results are expressed as average $\pm \mathrm{SD}$ or as median (IQR) for the bivariate analysis and as multivariable-adjusted average \pm SE for the multivariable analysis. Bivariate analysis performed using Student's t-test or Kruskal-Wallis non-parametric test (*).Multivariable analysis conducted using analysis of variance adjusting for gender, age group, BMI categories, insomnia categories, educational level, diabetes, presence of antihistame, antidepressant or hypnotic drugs, self-rated health, and depression. $\mathrm{BMI}$, body mass index; TSH, thyroid stimulating hormone.

fatigue. The comparison between included and excluded participants is provided in online supplementary table 1 , and the results of the multivariable analysis are provided in online supplementary table 2. Excluded participants were more frequently women, were older, had a lower educational level, were more frequently never or current smokers, had more comorbidities (cardiovascular diseases, diabetes, anaemia and hypertension) and rated their health worse.

\section{Prevalence and factors associated with fatigue}

The overall prevalence of fatigue as defined by an FSS score $\geq 4$ was $21.9 \%$ (95\% CI $20.4 \%$ to $23.4 \%$ ) and was higher in women at $23.4 \%$ (95\% CI $21.3 \%$ to $25.7 \%$ ) than in men at $20.1 \%$ (95\% CI $18.0 \%$ to $22.3 \%$ ) ( $\mathrm{p}=0.031)$. The distribution of an FSS score $\geq 5$ (prevalence of fatigue $10.9 \%$ ) is provided in online supplementary figure 2 ; the number of participants with fatigue decreased when the levels of FSS increased.

The analysis of the factors associated with fatigue as defined by an FSS score $\geq 4$ is provided in tables 1 and 2 .

On bivariate analysis, participants with fatigue were younger, had a higher BMI, a lower handgrip strength and lower ferritin levels (table 1). Participants with fatigue were more frequently women, had a lower educational level, and presented more frequently with clinical insomnia, diabetes, anaemia, depression and low TSH values (table 2). Finally, participants with fatigue had a higher consumption of antihistamines, antidepressants and hypnotics, and rated more frequently their health as bad or very bad (table 2).

Multivariable analysis showed that obesity (OR CI 1.40 (95\% CI 1.03 to 1.91$)$ ), insomnia categories ( $\mathrm{p}$ value for trend $<0.001$ ), depression (OR 3.26 (95\% CI 2.38 to $4.46)$ ), anaemia (OR 1.70 (95\% CI 1.00 to 2.89 )) and low self-rated health status ( $p$ value for trend $<0.001$ ) were positively associated, while older age ( $p$ value for trend 0.002) was negatively associated with fatigue. Conversely, no association was found for diabetes, TSH levels, antihistamines or hypnotics (table 2).

\section{Sensitivity analyses}

The overall prevalence of fatigue as defined by an FSS score $\geq 5$ was $10.9 \%$ (95\% CI $9.7 \%$ to $12.1 \%$ ) and was higher in women at $12.3 \%$ (95\% CI $10.7 \%$ to $14.0 \%$ ) than in men at $9.3 \%(95 \%$ CI $7.8 \%$ to $11.1 \%)(p=0.011)$. The results of the sensitivity analyses using an FSS threshold of $\geq 5$ are provided in online supplementary tables 3 and 4. Overall, the results were comparable with those using a threshold of $\geq 4$ : gender, insomnia categories ( $p$ value for trend $<0.001$ ) and low self-rated health status ( $p$ value for trend $<0.001$ ) were positively associated with fatigue. Conversely, no association was found for age, obesity, diabetes, TSH levels, antihistamines, antidepressants or hypnotics (online supplementary table 4).

Sensitivity analysis using inverse probability weighting by the propensity score led to similar findings, except that anaemia and antidepressants were no longer associated with fatigue, while a positive association was found between low TSH levels and fatigue (online supplementary table 5).

\section{DISCUSSION}

To our knowledge, this is one of the few studies assessing the prevalence and the factors associated with fatigue in a general population setting, and the first study conducted in Switzerland. Using an FSS cut-off of $\geq 4$, our results indicate that one out of five people aged between 45 and 86 years presents with fatigue, and that obesity, insomnia, depression and decreasing self-rated health status were positively associated with fatigue, while older age was negatively associated with fatigue. 
Table 2 Bivariate and multivariable analyses of the categorical factors associated with fatigue as defined by a Fatigue Severity Scale score $\geq 4$ in the Cohorte Lausannoise (CoLaus) study, Lausanne, Switzerland, 2014-2017

\begin{tabular}{|c|c|c|c|c|c|}
\hline \multirow{3}{*}{ Gender } & \multicolumn{3}{|l|}{ Bivariate } & \multicolumn{2}{|l|}{ Multivariable } \\
\hline & No fatigue & Fatigue & $P$ value & OR (95\% Cl) & $P$ value \\
\hline & & & 0.031 & & \\
\hline & $1066(47.9)$ & $268(43.0)$ & & 1 (ref) & \\
\hline Female & $1159(52.1)$ & $355(57.0)$ & & 1.25 (0.99 to 1.58 ) & 0.065 \\
\hline Age group & & & $<0.001$ & & \\
\hline $45-54$ & 643 (28.9) & $236(37.9)$ & & 1 (ref) & \\
\hline $55-64$ & $724(32.5)$ & $209(33.6)$ & & 0.69 (0.53 to 0.90$)$ & 0.006 \\
\hline $64-74$ & $626(28.1)$ & $113(18.1)$ & & 0.43 (0.31 to 0.59 ) & $<0.001$ \\
\hline $75+$ & $232(10.4)$ & 65 (10.4) & & 0.60 (0.40 to 0.90$)$ & 0.013 \\
\hline Educational level & & & 0.017 & & \\
\hline Primary & $249(11.2)$ & $93(14.9)$ & & 1 (ref) & \\
\hline Apprenticeship & $794(35.7)$ & $221(35.5)$ & & 1.05 (0.73 to 1.51$)$ & 0.782 \\
\hline High school & $626(28.1)$ & $182(29.2)$ & & 1.13 (0.78 to 1.64$)$ & 0.520 \\
\hline University & $556(25.0)$ & $127(20.4)$ & & 0.98 (0.66 to 1.46$)$ & 0.937 \\
\hline Smoking categories & & & 0.279 & & \\
\hline Never & 907 (41.7) & $242(39.7)$ & & - & \\
\hline Former & $866(39.8)$ & $264(43.4)$ & & - & \\
\hline Current & $402(18.5)$ & 103 (16.9) & & - & \\
\hline BMI categories & & & $<0.001$ & & \\
\hline Underweight & $37(1.7)$ & $5(0.8)$ & & 0.69 (0.24 to 2.01$)$ & 0.495 \\
\hline Normal & $920(41.4)$ & 219 (35.2) & & 1 (ref) & \\
\hline Overweight & $914(41.1)$ & $243(39.0)$ & & 1.01 (0.78 to 1.31 ) & 0.942 \\
\hline Obese & 354 (15.9) & $156(25.0)$ & & 1.40 (1.03 to 1.91$)$ & 0.032 \\
\hline Insomnia categories & & & $<0.001$ & & \\
\hline No insomnia & $1782(86.2)$ & 335 (62.6) & & 1 (ref) & \\
\hline Subthreshold & $233(11.3)$ & $114(21.3)$ & & 1.57 (1.16 to 2.13 ) & 0.003 \\
\hline Clinical insomnia & $53(2.6)$ & $86(16.1)$ & & 3.76 (2.41 to 5.86 ) & $<0.001$ \\
\hline Caffeinated drinks & & & 0.147 & & \\
\hline None & 205 (9.5) & 75 (12.3) & & - & \\
\hline $1-3 /$ day & $1418(65.5)$ & $374(61.5)$ & & - & \\
\hline $4-6 /$ day & $471(21.8)$ & $137(22.5)$ & & - & \\
\hline $7+/$ day & $70(3.2)$ & $22(3.6)$ & & - & \\
\hline Self-rated health & & & $<0.001$ & & \\
\hline Very good & 621 (27.9) & $58(9.3)$ & & 1 (ref) & \\
\hline Good & $1323(59.5)$ & $294(47.2)$ & & 1.94 (1.39 to 2.71 ) & $<0.001$ \\
\hline Average & $270(12.1)$ & $232(37.2)$ & & 5.55 (3.78 to 8.14$)$ & $<0.001$ \\
\hline Bad + very bad & $11(0.5)$ & $39(6.3)$ & & 14.1 (5.95 to 33.4 ) & $<0.001$ \\
\hline Cardiovascular disease & & & 0.697 & & \\
\hline No & $2036(91.5)$ & $567(91.0)$ & & - & \\
\hline Yes & $189(8.5)$ & $56(9.0)$ & & - & \\
\hline Diabetes & & & $<0.001$ & & \\
\hline No & 2069 (93.2) & 547 (87.9) & & 1 (ref) & \\
\hline Yes & $151(6.8)$ & 75 (12.1) & & 1.24 (0.82 to 1.87 ) & 0.306 \\
\hline Depression (CES-D) & & & $<0.001$ & & \\
\hline No & $2026(93.8)$ & $404(67.6)$ & & 1 (ref) & \\
\hline
\end{tabular}


Table 2 Continued

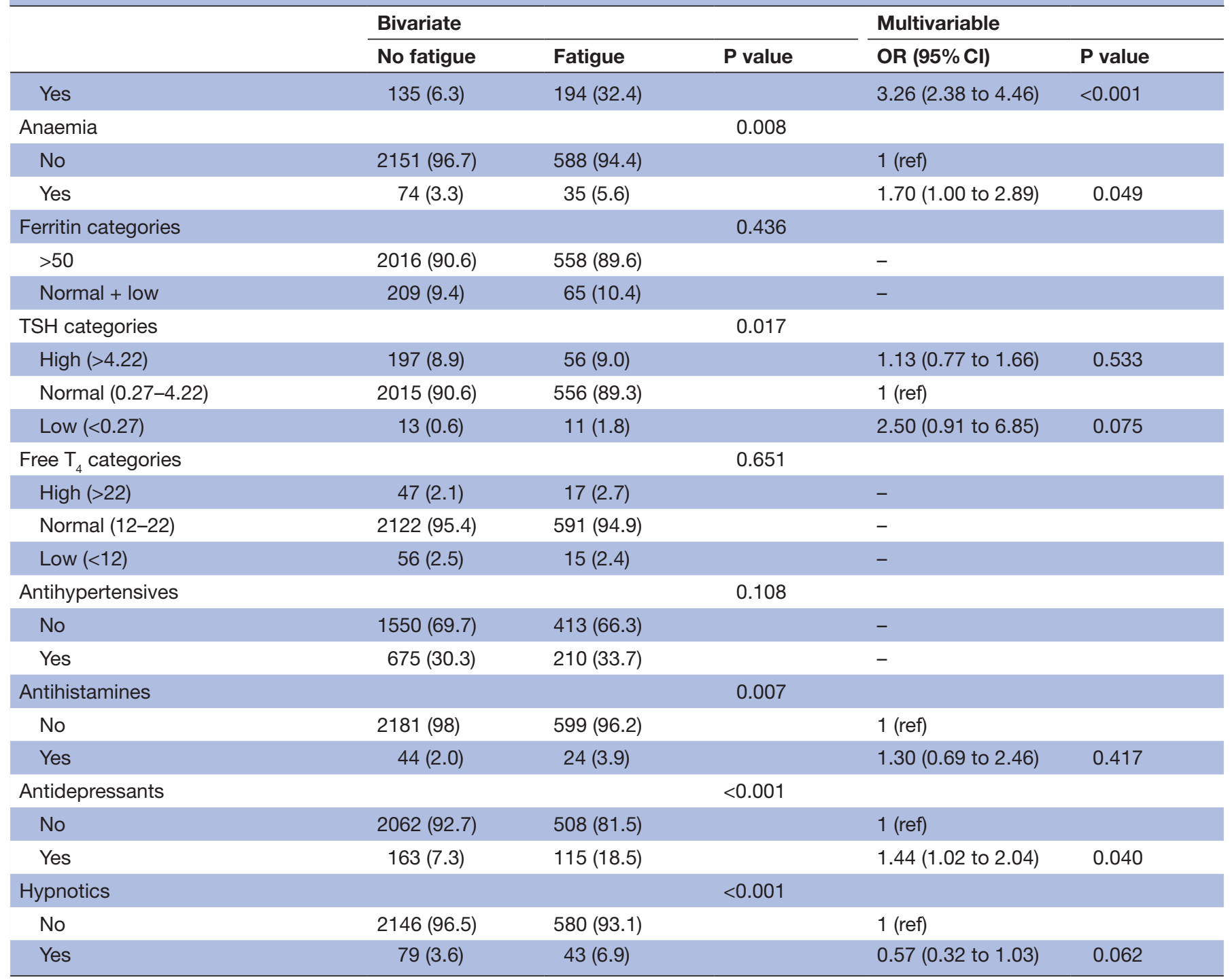

Results are expressed as the number of participants (row percentage) for the bivariate analysis and as multivariable-adjusted OR (95\% $\mathrm{Cl}$ ) for the multivariable analysis. Bivariate analysis performed using $\chi^{2}$; multivariable analysis performed using logistic regression. Only variables with $\mathrm{p}<0.05$ in the bivariate analysis were retained for the multivariable analysis.

-, not retained; BMI, body mass index; CES-D, Center for Epidemiologic Studies-Depression; TSH, thyroid stimulating hormone; ref, reference.

\section{Prevalence of fatigue}

Using the cut-off of $\geq 4$, fatigue was present in one out of five participants $(22.1 \%)$, a finding in agreement with the study by Loge et $a l^{8}{ }^{8}$ which reported a prevalence of 22\% among 2323 participants using the Chalder Fatigue Scale. Conversely, the cross-sectional study by Lerdal $e t$ $a l,{ }^{19}$ which used the FSS in a sample of 1893 participants, reported a prevalence of fatigue of $46.7 \%$ and $23.1 \%$ using a cut-off of $\geq 4$ and $\geq 5$, respectively, in comparison with $22.1 \%$ and $10.9 \%$ in our study. The investigated population was aged 19-81 years and included younger participants (women of childbearing age with menstruation and young parents), compared with our study which included participants aged between 45 and 86 years, which could explain this difference in the prevalence of fatigue. A study conducted in general practice reported a prevalence of fatigue of $38 \%$ using the Chalder Fatigue Scale ${ }^{2}$ whereas a study conducted in the Dutch working population reported a prevalence of fatigue of $22 \%$ using other fatigue measures. ${ }^{6}$ Comparison between studies is hampered by the small number of studies assessing the prevalence of fatigue in non-selected samples, the different fatigue scales used and the somewhat different settings (ie, general population vs general practice). Still, they provide a first basis for comparison, and it would be important that future studies use similar assessment methods to facilitate comparisons. Overall, our results suggest that the prevalence of fatigue in the Lausanne population is comparable or lower than reported previously. 


\section{Clinical and societal factors associated with fatigue}

Women tended to report fatigue more frequently than men, but this association was no longer significant after multivariable adjustment. Higher prevalence of fatigue in women has been found in some studies ${ }^{815}$ but not in others. ${ }^{18}$ In a Swedish study conducted in 2014, Engberg et $a l^{16}$ considered that this difference could be due to factors related to gender inequalities regarding household responsibilities and child raising, as the gender gap in general fatigue was largest among those aged $<55$ years.

Younger people reported fatigue more frequently than the elderly, a finding in agreement with a Swedish study conducted in $2014 .{ }^{16}$ Similarly, a previous study found that older subjects complain less of sleepiness. ${ }^{30}$ Still, this association was no longer statistically significant when the cut-off of $\geq 5$ was applied to define fatigue, suggesting that young subjects tend to present with borderline fatigue as suggested previously. ${ }^{19}$ Conversely, earlier studies (1990-2000) found a positive association between age and fatigue. ${ }^{81721}$ A possible explanation for this difference is that older people might have a better quality of life nowadays and are less depressed. Although there is little information regarding trends in quality of life among Swiss elderly, the "Vivre/Leben/Vivere" study ${ }^{31}$ concluded that the quality of life among Swiss elderly increased in the last 30 years. ${ }^{32}$ Indeed, in our study, the lowest prevalence of fatigue was reported by participants aged 64-74 years, which are the 'young' retired with few comorbidities. Similarly, the prevalence of depression was lower in elderly than in younger participants $(8.1 \%$ and $10.2 \%$ in the $65-74$ and the $75+$ years, respectively, vs $15.1 \%$ and $12.5 \%$ in the $45-54$ and $55-64$ years, respectively; $\mathrm{p}<0.001$ ).

Obese subjects had a higher prevalence of fatigue defined by an FSS score $\geq 4$. This finding is in agreement with studies conducted in the USA ${ }^{33}$ and in the UK. ${ }^{13}$ Still, this association was no longer statistically significant when the cut-off of $\geq 5$ was applied to define fatigue, suggesting that obese subjects tend to present with borderline fatigue as suggested previously. ${ }^{19}$ Obesity is a risk factor for sleep apnoea, which leads to increased daytime sleepiness. Still, the association persisted after adjusting for insomnia, a finding in agreement with a previous study that showed that obese subjects have excessive fatigue independently of sleep-disordered breathing. ${ }^{34}$ Because it excluded too many subjects, we did not correlate obesity and sleep-disordered breathing in our study. A possible explanation could be the increase in proinflammatory cytokines in obese subjects, ${ }^{35}$ which would lead to higher fatigue, ${ }^{36}$ but other factors such as decreased physical fitness should be further explored.

A positive association was found between self-reported clinical insomnia and fatigue, and this association was independent of obesity, depression and antidepressant medication. Fatigue is a core symptom of insomnia, ${ }^{34}$ and a Norwegian study conducted in 2014 showed that reducing insomnia severity led to a concomitant reduction in fatigue. ${ }^{37}$ Interestingly, many subjects with sleep complaints do not consult for this issue, ${ }^{38}$ which might lead to an underestimation of its prevalence. Overall, our results suggest that insomnia is an important and underestimated factor of fatigue.

Both depression and antidepressant medication were independently and positively associated with fatigue. The association between depression and fatigue has been repeatedly reported, ${ }^{13} 39-41$ and the same applies for antidepressant medication. ${ }^{3}$ Our results confirm the known association between depression and fatigue, and suggest that antidepressant treatment might not systematically relieve fatigue among subjects with depression. Furthermore, fatigue is a common side effect of antidepressant therapy and a symptom of depression, making the identification of the cause of fatigue difficult with a possibility of reverse causality (fatigue leading to depression and vice versa). We used a one-dimensional tool to evaluate fatigue (FSS); hence, we cannot distinguish between physical and mental fatigue. There is considerable overlap in the phenomenology of fatigue and depression or anxiety, but there are some important differences. People with fatigue without psychiatric symptoms tend to attribute their symptoms to external causes, while most depressed people experience self-blame or lowered self-esteem. ${ }^{42}$ Further, fatigue and depression commonly appear together. A study conducted in 2009 by Harvey et $a t^{43}$ showed that $7 \%$ of fatigued persons have no psychiatric symptoms, but remain at increased risk of later psychiatric disorder independently of the severity of fatigue.

A strong association was found between poor self-rated health and fatigue, a finding also reported elsewhere. ${ }^{616}$ Low self-rated health has been associated with increased levels of inflammatory markers such as interleukin 6 and $\mathrm{CRP},{ }^{44}$ which in turn could trigger fatigue. Conversely, increased fatigue might lead to a lower rating of health status.

\section{Biological factors associated with fatigue}

Participants with anaemia had a higher likelihood of reporting fatigue. This finding is in agreement with the literature, ${ }^{45}$ although no association between fatigue and low haemoglobin levels was found in a UK study. ${ }^{13}$ A possible explanation is that in the UK study, anaemia was defined as a haemoglobin $<110 \mathrm{~g} / \mathrm{L}$, which is lower than the thresholds used in our study $(<133 \mathrm{~g} / \mathrm{L}$ for men and $<117 \mathrm{~g} / \mathrm{L}$ for women). This led to a small sample size (356 participants, corresponding to $1.9 \%$ of the overall sample) and thus a low statistical power.

Hypothyroidism is often cited during the investigation of fatigue. ${ }^{10}$ In this study, participants with low TSH levels reported fatigue more frequently, but this association was significant only after multivariable analysis with inverse probability weighting. Furthermore, the prevalence of low TSH levels was $<1 \%$ in the overall sample. The associations between hypothyroidism and fatigue have been controversial for a long time. ${ }^{10}$ Basu et $\mathrm{l}^{13}$ found no association between TSH categories and fatigue, and Canaris $e t$ $a l^{47}$ reported that the association between hypothyroidism 
and fatigue was weak. Overall, our results suggest that, in the presence of fatigue, hypothyroidism is an unlikely cause and should not be systematically assessed.

\section{Implications of the study}

Based on our study findings, we propose to focus on specific clinical and biological factors amenable to treatment at an individual level. Regarding clinical factors, sleep disturbances such as insomnia and sleep apnoea (namely in the presence of a patient with obesity) and the presence of depression should be assessed. Lifestyle measures to improve sleep quality and quantity should be preferred to medication. ${ }^{22}$ In the case of depression, it will be important to warn patients that antidepressant medication might not necessarily lead into rapid relief of fatigue. Regarding biological factors, anaemia should be ruled out, while screening for hypothyroidism is not recommended as a first step.

At the population level, preventive measures such as stress management and health promotion like relaxation, time management and cognitive reframing (eg, within the work environment) could improve sleep quality, increase self-rated health ${ }^{48}$ and consequently reduce fatigue.

\section{Strengths and limitations}

This study has several strengths. First, it is one of the few studies assessing the prevalence and the factors associated with fatigue in a population-based sample, which is of interest for public health. Second, it explored a large panel of possible factors associated with fatigue, thus allowing the identification of factors significantly and independently associated with fatigue.

This study has also several limitations. First, its cross-sectional setting precludes the identification of the causes of fatigue, as reverse causality is possible (ie, fatigue leading to depression and vice versa). ${ }^{3}$ All participants of the CoLaus study are currently being recontacted and re-examined, so a prospective analysis of the causes of fatigue will be feasible within 2 years. Second, there is no gold standard for the evaluation of fatigue and no official definition of fatigue. Hence, results might vary according to the scale applied or how participants interpret the term 'fatigue'. In this study, we chose to use a scale that was previously applied by other authors to facilitate comparisons. Third, only the German version of the FSS has been validated in Switzerland; the French version used in this study has not yet been validated. Hence, it is possible that the true prevalence levels of fatigue might be underestimated or overestimated, or that some items of the questionnaire might not be informative. Still, the Cronbach's alpha for the questionnaire was 0.918, suggesting an excellent internal consistency. Furthermore, our results provide a first estimation of the prevalence of fatigue in the Swiss French-speaking general population, which could serve as a reference for further studies. Fourth, a sizeable fraction of the sample was excluded, both between the baseline and the second follow-up, and within the current study, which might limit the generalisability of the findings. For instance, excluded participants were more frequently women; as women reported more frequently fatigue, this might lead to an underestimation of prevalence rates or a decrease in the strength of the associations. Still, an analysis using a propensity score weighting for the probability of being excluded led to similar findings. Conversely, it was not possible to assess the reasons why participants did not complete the questionnaire. Fifth, no information was available regarding shift work or the presence of very young children. Still, as a sizeable fraction (almost $70 \%$ ) of the sample was aged over 55 and over $36 \%$ of the sample was aged over 64 , it is likely that the number of participants either on shift work or with very young children would be small. Sixth, the FSS explored fatigue during the previous week, while the ISI score explored sleep during the previous month. Hence, it is possible that the time association between the two variables might not be optimal. Still, as the FSS lies within the period encompassed by the ISI, we believe that the associations obtained are clinically relevant. Seventh, the study is limited to the population of aged 45-86, and its generalisability remains to be assessed. For instance, no information was collected regarding other confounders among younger subjects, where prevalence of fatigue might be higher due to parental and professional duties. ${ }^{49}$ Finally, possible biases related to the self-reporting of fatigue could not be avoided, such as overestimation or underestimation of symptoms or misunderstanding of what the term 'fatigue' meant; still, this dilution bias would lead to a decrease in the strength of the associations, and it would be too restrictive in our opinion to provide a definition of the term 'fatigue' to the participants, as different interpretations of the definition itself could also occur.

\section{Recommendations for future studies}

Future studies on the prevalence of fatigue in the general population should focus on the following topics: (1) validate the questionnaires in the population of interest; and (2) whenever possible, use a standardised questionnaire to allow comparison between studies.

While some factors such as obesity, ${ }^{133}$ depression ${ }^{13} 39-41$ and antidepressant medications ${ }^{3}$ were consistently associated with fatigue in our study and in the literature, controversial findings such as the association between fatigue and gender, age groups and anaemia should be further explored.

\section{CONCLUSION}

In a population-based sample aged 45-86, fatigue was present in one out of five subjects. Regarding clinical factors, sleep disturbances such as insomnia and sleep apnoea should be assessed first, followed by depression. Regarding biological factors, anaemia should be ruled out, while screening for hypothyroidism is not recommended as a first step. Sleep complaints and fatigue in older subjects are not due to ageing and should prompt the identification of the underlying cause. 
Contributors CG conducted the literature search, interpreted the results and wrote the manuscript. PM-V performed the statistical analyses and wrote part of the manuscript. PV conceived the study and revised the manuscript for important intellectual content. PM-V has full access to the data and is the guarantor of the manuscript. All authors have read and approved this version of the manuscript.

Funding The CoLaus study was and is supported by research grants from GlaxoSmithKline, the Faculty of Biology and Medicine of Lausanne, and the Swiss National Science Foundation (grants 33CSC0-122661, 33CS30-139468 and $33 \mathrm{CS} 30-148401)$. The funding sources had no involvement in the study design, data collection, analysis and interpretation, writing of the report, or decision to submit the article for publication.

\section{Competing interests None declared.}

Patient consent for publication Not required.

Ethics approval The Institutional Ethics Committee of the University of Lausanne, which afterwards became the Ethics Commission of Canton Vaud (www.cer-vd. ch), approved the baseline CoLaus study (reference 16/03); the approval was renewed for the first (reference 33/09) and the second (reference 26/14) follow-up. The full decisions of the CER-VD can be obtained from the authors on request. The study was performed in agreement with the Helsinki Declaration and its former amendments, and in accordance with the applicable Swiss legislation. All participants gave their signed informed consent before entering the study.

Provenance and peer review Not commissioned; externally peer reviewed.

Data availability statement Data may be obtained from a third party and are not publicly available. All data relevant to the study are included in the article or uploaded as supplementary information.

Open access This is an open access article distributed in accordance with the Creative Commons Attribution Non Commercial (CC BY-NC 4.0) license, which permits others to distribute, remix, adapt, build upon this work non-commercially, and license their derivative works on different terms, provided the original work is properly cited, appropriate credit is given, any changes made indicated, and the use is non-commercial. See: http://creativecommons.org/licenses/by-nc/4.0/.

\section{REFERENCES}

1. Mota DDCF, Pimenta CAM. Self-report instruments for fatigue assessment: a systematic review. Res Theory Nurs Pract 2006;20:49-78.

2. Pawlikowska T, Chalder T, Hirsch SR, et al. Population based study of fatigue and psychological distress. BMJ 1994;308:763-6.

3. Guessous I, Favrat B, Cornuz J, et al. [Fatigue: review and systematic approach to potential causes]. Rev Med Suisse 2006;2:2725-31.

4. Lewis G, Wessely S. The epidemiology of fatigue: more questions than answers. J Epidemiol Community Health 1992;46:92-7.

5. Fuhrer R, Wessely S. The epidemiology of fatigue and depression: a French primary-care study. Psychol Med 1995;25:895-905.

6. Bültmann U, Kant I, KasI SV, et al. Fatigue and psychological distress in the working population: psychometrics, prevalence, and correlates. J Psychosom Res 2002;52:445-52.

7. Dittner AJ, Wessely SC, Brown RG. The assessment of fatigue: a practical guide for clinicians and researchers. J Psychosom Res 2004;56:157-70.

8. Loge JH, Ekeberg O, Kaasa S. Fatigue in the general Norwegian population: normative data and associations. J Psychosom Res 1998;45:53-65.

9. Krupp LB, LaRocca NG, Muir-Nash J, et al. The fatigue severity scale. Application to patients with multiple sclerosis and systemic lupus erythematosus. Arch Neurol 1989;46:1121-3.

10. Cornuz J, Guessous I, Favrat B. Fatigue: a practical approach to diagnosis in primary care. Can Med Assoc J 2006;174:765-7.

11. Reid S, Chalder T, Cleare A, et al. Chronic fatigue syndrome. BMJ 2000;320:292-6

12. Cullen W, Kearney $Y$, Bury G. Prevalence of fatigue in general practice. Ir J Med Sci 2002;171:10-12.

13. Basu N, Yang X, Luben RN, et al. Fatigue is associated with excess mortality in the general population: results from the EPIC-Norfolk study. BMC Med 2016;14:122.

14. Bates DWet al. Prevalence of fatigue and chronic fatigue syndrome in a primary care practice. Arch Intern Med 1993;153:2759-65.

15. Fieo RA, Mortensen EL, Lund R, et al. Assessing fatigue in latemidlife: increased scrutiny of the multiple fatigue Inventory-20 for community-dwelling subjects. Assessment 2014;21:706-12.

16. Engberg I, Segerstedt J, Waller G, et al. Fatigue in the general population- associations to age, sex, socioeconomic status, physical activity, sitting time and self-rated health: the Northern Sweden MONICA study 2014. BMC Public Health 2017;17:654.

17. Watt Tet al. Fatigue in the Danish general population. Influence of sociodemographic factors and disease. J Epidemiol Community Health 2000;54:827-33.

18. David A, Pelosi A, McDonald E, et al. Tired, weak, or in need of rest: fatigue among general practice attenders. BMJ 1990;301:1199-202.

19. Lerdal A, Wahl AK, Rustoen T, et al. Fatigue in the general population: a translation and test of the psychometric properties of the Norwegian version of the fatigue severity scale. Scand J Public Health 2005;33:123-30.

20. Boter H, Mänty M, Hansen AM, et al. Self-Reported fatigue and physical function in late mid-life. J Rehabil Med 2014;46:684-90.

21. Schwarz R, Krauss O, Hinz A. Fatigue in the general population. Onkologie 2003;26:140-4.

22. Firmann M, Mayor V, Vidal PM, et al. The CoLaus study: a population-based study to investigate the epidemiology and genetic determinants of cardiovascular risk factors and metabolic syndrome. BMC Cardiovasc Disord 2008;8:6.

23. Valko PO, Bassetti CL, Bloch KE, et al. Validation of the fatigue severity scale in a Swiss cohort. Sleep 2008;31:1601-7.

24. Laranjeira CA. Translation and adaptation of the fatigue severity scale for use in Portugal. Appl Nurs Res 2012;25:212-7.

25. Bastien $\mathrm{CH}$, Vallières $\mathrm{A}$, Morin $\mathrm{CM}$. Validation of the insomnia severity index as an outcome measure for insomnia research. Sleep Med 2001;2:297-307

26. Lenore SR. The CES-D scale: a self-report depression scale for research in the general population. Applied Psychological Measurement 1977;1:385-401.

27. Fuhrer R, Rouillon F. La version Française de l'échelle CES-D: description et traduction de l'échelle d'auto-évaluation. Psychiatry and Psychobiology 1989;4:163-6.

28. Hamieh N, Meneton P, Wiernik E, et al. Depression, treatable cardiovascular risk factors and incident cardiac events in the Gazel cohort. Int J Cardiol 2019;284:90-5.

29. Austin PC. An introduction to propensity score methods for reducing the effects of confounding in observational studies. Multivariate Behav Res 2011;46:399-424.

30. Luca G, Haba Rubio J, Andries D, et al. Age and gender variations of sleep in subjects without sleep disorders. Ann Med 2015;47:482-91.

31. Ludwig C, Cavalli S, Oris M. "Vivre/Leben/Vivere": An interdisciplinary survey addressing progress and inequalities of aging over the past 30 years in Switzerland. Arch Gerontol Geriatr 2014;59:240-8.

32. edslisted noauthors. Qualité de vie des seniors en Suisse. In: Centre interfacultaire de gérontologie et d'études des vulnérabilités, Pôle de Recherche national lives. Geneva, Switzerland: University of Geneva, 2015.

33. Resnick HE, Carter EA, Aloia M, et al. Cross-Sectional relationship of reported fatigue to obesity, diet, and physical activity: results from the third National health and nutrition examination survey. J Clin Sleep Med 2006;2:163-9.

34. Bixler EO, Vgontzas AN, Lin H-M, et al. Excessive daytime sleepiness in a general population sample: the role of sleep apnea, age, obesity, diabetes, and depression. J Clin Endocrinol Metab 2005;90:4510-5.

35. Marques-Vidal P, Bochud M, Bastardot F, et al. Association between inflammatory and obesity markers in a Swiss population-based sample (CoLaus study). Obes Facts 2012;5:734-44.

36. Vgontzas AN, Bixler EO, Chrousos GP. Obesity-related sleepiness and fatigue: the role of the stress system and cytokines. Ann NY Acad Sci 2006;1083:329-44.

37. Kallestad $\mathrm{H}$, Jacobsen $\mathrm{HB}$, Landrø $\mathrm{NI}$, et al. The role of insomnia in the treatment of chronic fatigue. J Psychosom Res 2015;78:427-32.

38. Pires MLN, Benedito-Silva AA, Mello MT, et al. Sleep habits and complaints of adults in the city of São Paulo, Brazil, in 1987 and 1995. Braz J Med Biol Res 2007;40:1505-15.

39. Lawrie SM, Manders DN, Geddes JR, et al. A population-based incidence study of chronic fatigue. Psychol Med 1997;27:343-53.

40. Wessely S, Chalder T, Hirsch S, et al. Psychological symptoms, somatic symptoms, and psychiatric disorder in chronic fatigue and chronic fatigue syndrome: a prospective study in the primary care setting. Am J Psychiatry 1996;153:1050-9.

41. Skapinakis $P$, Lewis $G$, Meltzer $H$. Clarifying the relationship between unexplained chronic fatigue and psychiatric morbidity: results from a community survey in Great Britain. Int Rev Psychiatry 2003;15:57-64.

42. Powell R, Dolan R, Wessely S. Attributions and self-esteem in depression and chronic fatigue syndromes. J Psychosom Res 1990;34:665-73.

43. Harvey SB, Wessely S, Kuh D, et al. The relationship between fatigue and psychiatric disorders: evidence for the concept of neurasthenia. J Psychosom Res 2009;66:445-54. 
44. Christian LM, Glaser R, Porter K, et al. Poorer self-rated health is associated with elevated inflammatory markers among older adults. Psychoneuroendocrinology 2011;36:1495-504.

45. Cella D, Eton DT, Lai J-S, et al. Combining anchor and distributionbased methods to derive minimal clinically important differences on the functional assessment of cancer therapy (fact) anemia and fatigue scales. J Pain Symptom Manage 2002;24:547-61.

46. Cella D, Lai J-shei, Chang $\mathrm{C}-\mathrm{H}$, et al. Fatigue in cancer patients compared with fatigue in the general United States population. Cancer 2002;94:528-38.
47. Canaris GJ, Manowitz NR, Mayor G, et al. The Colorado thyroid disease prevalence study. Arch Intern Med 2000;160:526-34.

48. Hasson D, Arnetz BB, Theorell T, et al. Predictors of self-rated health: a 12-month prospective study of it and media workers. Popul Health Metr 2006;4:8.

49. Bensing JM, Hulsman RL, Schreurs KM. Gender differences in fatigue: biopsychosocial factors relating to fatigue in men and women. Med Care 1999;37:1078-83. 